ARTICLE

\title{
Evaluation of the CANDU 6 Neutron Characteristics in View of Application of the Resonance Dependent Scattering Kernel in MCNP(X)
}

\author{
Ron DAGAN $^{1, *}$, Björn BECKER ${ }^{2}$ and Dan ROUBTSOV ${ }^{3}$ \\ ${ }^{1}$ Institute for Neutron Physics and Reactor Technology (INR), Forschungszentrum Karlsruhe GmbH, \\ Postfach 3640, D-76021 Karlsruhe, Germany \\ ${ }^{2}$ Department of Mechanical, Aerospace, and Nuclear Engineering, Rensselaer Polytechnic Institute, \\ Troy, New York 12180, USA \\ ${ }^{3}$ Atomic Energy of Canada Limited, Chalk River Laboratories, Chalk River, ON, K0J 1J0, Canada
}

\begin{abstract}
This study continues our investigation of the influence of the resonant scattering kernel on different reactor types and, in particular, different moderators and coolants. The importance of an advanced neutron scattering treatment for heavy nuclei with strong energy-dependent cross sections, such as the pronounced resonances of ${ }^{238} \mathrm{U}$, has been discussed in various publications, where the impact of the full double-differential scattering kernel on the core characteristics was derived. In this study, we concentrate on the application of the resonant dependent kernel to heavy water reactors, namely, the CANDU ${ }^{\circledR} 6$.

In modeling nuclear reactors with Monte Carlo methods, we take advantage of the stochastic implementation of the resonant scattering kernel directly in $\operatorname{MCNP}(\mathrm{X})$, the so-called Doppler Broadening Rejection Correction - DBRC, which allows the direct calculation of the differential part of the Doppler broadened cross section. The DBRC model is based on an additional rejection scheme within the procedure "sampling of the target velocity" in the subroutine tgtvel in MCNP(X). This means that the DBRC samples the Doppler broadening of the double-differential cross section "on-line" and consistently for each neutron undergoing a scattering interaction with a heavy nuclide.

The introduction of the resonant scattering kernel within postulated modes of the CANDU 6 lattice cell simulation leads to a predicted decrease in criticality of about $50 \mathrm{pcm}(=0.5 \mathrm{mk})$ near the design conditions and up to 100 $150 \mathrm{pcm}(=1.0-1.5 \mathrm{mk})$ at higher fuel temperatures. The standard deviation of these estimates is $8 \mathrm{pcm}(=0.08 \mathrm{mk})$. We predict a decrease in the fuel temperature coefficient of reactivity (Doppler Effect) by $\sim 3$ to $10 \%$ for fresh clean fuel. We found no noticeable effect of the DBRC on the coolant void reactivity coefficient, the difference being less than $10 \mathrm{pcm}(=0.1 \mathrm{mk})$ near the design conditions. In addition, some artificial lattice cells were simulated showing that, by decreasing the cell pitch, the impact of the new resonant scattering kernel predictions increases significantly.
\end{abstract}

KEYWORDS: resonant scattering kernel, Doppler broadening rejection correction, DBRC, MCNP, MCNPX, CANDU

\section{Introduction}

The development of the full double-differential resonant scattering kernel by Rothenstein and Dagan ${ }^{1)}$ and the further implementation of this kernel within the NJOY $\operatorname{code}^{2)}$ by Rothenstein $^{3)}$ allowed the improved treatment of the secondary energy distribution with Monte Carlo codes. Dagan ${ }^{4)}$ generalized the use of probability $S(\alpha, \beta)$ tables, which were commonly used previously only for light nuclei, to model neutron interactions with heavy nuclei, in particular in the vicinity of their pronounced low-lying resonances. The effect on criticality, Doppler broadening, and fuel inventory turned out to be significant for light water reactors and also for high-temperature gas-cooled reactors. ${ }^{5,6)}$

In a later study by Becker et al., ${ }^{7}$ it was shown that a stochastic approach, namely, Doppler Broadening Rejection Correction (DBRC) based on an idea of Rothenstein, ${ }^{8}$ can be used to replace the above mentioned probability tables for heavy nuclei. This new algorithm scheme allows, to some

*Corresponding author, E-mail: dagan@inr.fzk.de extent, a simplification of the use of the double differential scattering kernel, and no precautions are needed for the guarantee of the model accuracy.

Strictly speaking, the idea of Rothenstein ${ }^{8)}$ was to use the existing scheme of MCNP, known as "sampling of the target velocity" to sample the secondary energy distribution. However, the approximation of energy independent cross section has to be replaced by the correct resonance treatment via an additional rejection-based sampling within the existing algorithm. The validity of this approach was further confirmed by dedicated experiments performed at the LINAC facility at the Rensselaer Polytechnic Institute by Danon et al. ${ }^{9)}$

Monte Carlo codes such as $\mathrm{MCNP}^{10)}$ or $\mathrm{MCNPX}^{11)}$ are widely accepted as the best available reference for neutron physics analysis. In view of this fact, it is of interest to directly compare the physical effects of the new scattering model for heavy nuclei embedded in $\mathrm{MCNP}^{7)}$ with the standard energy-independent scattering kernel of MCNP in a simple and efficient manner, as was suggested by Dagan. ${ }^{4)}$

In general, a need for high accuracy in the design of new types of reactors with unique types of fuel cells and different 
types of moderator and coolant, (e.g., heavy water and light water) calls for extensive investigations of the impact of the new resonant kernel on core characteristics. In this study, we quantify the effect of the new scattering approach in MCNP for both standard and specific innovative CANDU® reactor fuel types. Furthermore, the sensitivity of the resonant scattering kernel dependent parameters to changes of the core design is discussed.

\section{Energy Dependent Scattering Kernel}

The scattering kernel term within the transport equation $\sigma_{n n}^{T}\left(E \rightarrow E^{\prime}, \Omega \rightarrow \Omega^{\prime}\right)$ describes the source term of neutrons at energy $E^{\prime}$ in a specific control volume, based on the probability of neutrons scattered from another energy $E$ to $E^{\prime}$ and from a spatial direction $\Omega$ to $\Omega^{\prime}$.

The existing scattering kernel treatment in the Monte Carlo code MCNP is based either on the light nuclei model by Wigner-Wilkins ${ }^{12)}$ or on the asymptotic $0 \mathrm{~K}$ treatment for all materials. The asymptotic scattering kernel based on the basic two-body collision laws is well known: ${ }^{13)}$

$$
\begin{aligned}
& \frac{E^{\prime}}{E}=\frac{A^{2}+2 A \mu_{C M}+1}{(A+1)^{2}}, \\
& \mu_{l a b}^{0 K}=\frac{A \mu_{C M}+1}{\left(A^{2}+2 A \mu_{C M}+1\right)^{1 / 2}} .
\end{aligned}
$$

Here, $\mu_{C M}$ is the polar scattering angle in the center-ofmass frame, $\mu_{l a b}$ is the polar scattering angle in the laboratory frame and $A$ is the ratio of the atomic mass of the scattering nucleus to the neutron mass. $E$ and $E^{\prime}$ are the incident and secondary neutron energy, respectively. As can be seen this treatment omits the contribution of resonances as well as the effect of temperature.

For heavy nuclei with pronounced temperature dependent resonances, this method is insufficient and obviously leads to inconsistencies between the total scattering cross section and its differential part, namely, the scattering kernel. Traditionally, the total scattering cross section is Doppler broadened based on the relevant temperature and size of the resonances while the scattering kernel is not broadened in a consistent way.

A new double-differential scattering kernel developed by Rothenstein and Dagan ${ }^{1,3)}$ for heavy nuclei improves the existing kernel by introducing an energy-dependent cross section $\sigma_{s}(E)$ (Eq. (3)) within the integral of Eq. (4). In contrast, in the temperature-dependent probability scheme known as $\mathrm{S}(\alpha, \beta)$ tables $^{14)}$, the cross section $\sigma_{s}$ is taken to be a constant. Here, Eq. (3) displays the form that was included in a model used in the THERMR module of the NJOY code. $^{2)}$ (The THERMR module of NJOY 99 can be used to prepare the $S(\alpha, \beta)$ tables for heavy nuclei with pronounced resonances. ${ }^{4)}$ )

Note that the inclusion of the energy-dependent cross section term

CANDU $^{\circledR}$ (CANada Deuterium Uranium) is a registered trademark of Atomic Energy of Canada Limited (AECL)

$$
\left(\sigma_{s}^{t a b}\left[\left(\frac{A+1}{A^{2}}\right) \frac{(\xi+\tau)^{2}}{4} k_{B} T, 0\right]\right)
$$

is achieved by using the integral over the dimensionless velocities ( $\xi$ and $\tau$ ), and the dependency of the cross section on the temperature $T$ appears explicitly as well,

$$
\begin{aligned}
& \sigma_{s}^{T}(E \rightarrow E, \Omega \rightarrow \Omega)= \\
& \frac{1}{4 \pi E} \sqrt{\frac{A+1}{A \pi} \int_{\varepsilon_{\max }}^{\infty} d \xi \int_{\tau_{0}(\xi)}^{\tau_{1}(\xi)} d \tau\left(\frac{\xi+\tau}{2}\right)} \\
& \cdot\left(\sigma_{s}^{\text {tab }}\left[\left(\frac{A+1}{A^{2}}\right) \frac{(\xi+\tau)^{2}}{4} k_{B} T, 0\right]\right) \\
& \cdot \exp \left(v^{2}\left[\frac{(\xi+\tau)^{2}}{4 A}+\frac{(\xi-\tau)^{2}}{4}\right]\right) \\
& \cdot\left(\frac{\varepsilon_{\max } \varepsilon_{\min }(\xi-\tau)^{2}}{B_{0} \sin (\hat{\varphi})}\right)
\end{aligned}
$$

In Eq. (3), the superscript tab of the cross section indicates that it is a tabulated function, such as on a PENDF file, with specified (usually linear) interpolation laws between the successive entries. The data must be at $0 \mathrm{~K}$, as indicated by the second argument. In addition, one must further take into account that $\sigma_{s}^{t a b}$ is the bound atom scattering cross section and includes the factor $((A+1) / A)^{2}$ in its definition. In Eq. (4), the integration over the variables $\xi$ and $\tau$ replaces the integration over the velocity variables $t$ and $x$ where:

$$
t=u \sqrt{(A+1)} ; x=c \sqrt{(A+1)} .
$$

In addition,

$$
\varepsilon=v \sqrt{(A+1)} ; \varepsilon^{\prime}=v^{\prime} \sqrt{(A+1)}
$$

where $v$ and $v^{\prime}$ are the velocities of the neutron before and after the interaction, respectively. The velocity of the centreof-mass is $c$ and the velocity of the neutron in the centre-ofmass frame is $u$. $\varepsilon_{\max }$ and $\varepsilon_{\min }$ in Eq. (4) are the larger and the smaller value of $\varepsilon$ and $\varepsilon^{\prime}$, respectively. $\varphi$ is the azimuth angel and $\mathrm{B}_{0}$ is a velocity dependent parameter defined in the paper of Rothenstein. ${ }^{3)}$

The introduction of the variables $\xi$ and $\tau$ allows for an essential simplification of the mathematical algorithm for computing the double-differential scattering kernel. In particular, a quartic equation in $\tau$ that is based on the definition of parameter $B_{0}$ in Eq. (4) can be replaced by a bi-quadratic equation, as was shown in the paper of Rothenstein ${ }^{3)}$ to which we refer the reader for further information and explanation.

In modeling nuclear reactors with Monte Carlo methods, we take advantage of the stochastic implementation of the resonant scattering kernel directly in $\operatorname{MCNP}(\mathrm{X})$ that we call the Doppler Broadening Rejection Correction (DBRC) model. The DBRC model is based on an additional rejection scheme within the procedure "sampling of the target velocity" in the subroutine tgtvel in $\operatorname{MCNP}(\mathrm{X})$. This means that the DBRC samples the Doppler broadening of the doubledifferential cross section "on-line" and consistently for each neutron undergoing a scattering interaction with a heavy nuclide. Thus, it allows the direct calculation of the differential part of the Doppler broadened cross section for a given 


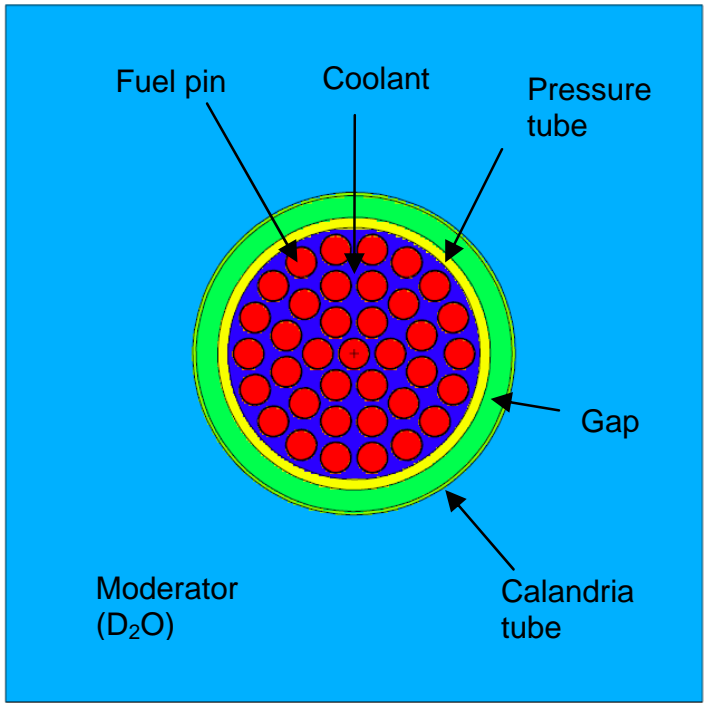

Fig. 1 37-Element CANDU 6 fuel bundle and lattice cell

nuclide at a given temperature.

The stochastic DBRC method is physically equivalent to the introduction of generalized scattering probability $\mathrm{S}(\alpha, \beta)$ tables for heavy isotopes, ${ }^{4)}$ and such tables were used as a reference solution for the verification of the stochastic method, before using it in the current study. However, in the stochastic approach, one has to prepare only the $0 \mathrm{~K}$ cross sections for the nuclei of interest $\left({ }^{238} \mathrm{U}\right.$ in this study), instead of generating a huge number (more than 1,500) of tables based on the analytic model, at a cost of about $15 \%$ increase in computer time of the MCNP run.

Further information and explanation on the practical aspects of implementation of the DBRC can be found on the NEA website. ${ }^{15)}$

\section{Impact of the DBRC Model on Criticality and Doppler Effect}

The CANDU reactor type is unique, because of its heavy water moderator. Consequently the core design is different from other reactor types, and so the impact of the resonance scattering kernel for this type of reactor should be different from LWRs or HTGRs - these were investigated previously.

In the following we concentrate mainly on the specific fuel bundle design used in CANDU 6. In addition, we test the sensitivity of the resonance scattering kernel to changes in the coolant and lattice pitch in view of the innovative CANDU concepts such as CANDU-SCWR, etc. ${ }^{16)}$

The analysis of CANDU reactors is based on a standard freshly fuelled 37-element CANDU bundle and a fuel lattice cell (see Fig. 1). The geometrical and material specifications of the bundle used are given elsewhere. ${ }^{1718)}$ The reference case bundle consists of natural uranium dioxide fuel, cooled and moderated by $\mathrm{D}_{2} \mathrm{O}$ of high (reactor grade) purity. For simplicity, all the fuel pins are assumed to have the same temperature. The numerical simulation is repeated for several fuel temperatures varying from $500 \mathrm{~K}$ to $2,000 \mathrm{~K}$, while all other temperatures are chosen according to Table 1.

In order to evaluate the impact of the resonance scattering kernel, each calculation was performed twice: first, with the
Table 1 Temperatures of CANDU 6 cell components

\begin{tabular}{ccc}
\hline Material & $\begin{array}{c}\text { Temperature } \\
{[\mathrm{K}]}\end{array}$ & $\begin{array}{c}\text { Temperature } \\
{[\mathrm{MeV}]}\end{array}$ \\
\hline Coolant & 573.6 & $4.943 \mathrm{E}-08$ \\
Moderator & 323.6 & $2.789 \mathrm{E}-08$ \\
Void (Gap) & 440.0 & $3.792 \mathrm{E}-08$ \\
Calandria tube & 340.0 & $2.930 \mathrm{E}-08$ \\
Pressure tube & 573.6 & $4.943 \mathrm{E}-08$ \\
Clad (sheath) & 573.6 & $4.943 \mathrm{E}-08$ \\
\hline
\end{tabular}

standard MCNPX (version 2.6f), and, second, with a modified MCNPX in which the DBRC method was introduced for ${ }^{238} \mathrm{U}$ up to $E=210 \mathrm{eV}$. This energy range covers the first eight most important $s$-wave resonances of ${ }^{238} \mathrm{U}$. The multi-temperature cross section library used is based on the JEFF-3.1 evaluated nuclear data library.

The Doppler reactivity coefficient is determined by fitting an equation of type:

$$
k_{\infty}\left(T_{f}\right) \approx a+b T_{f}^{1 / 2}+d T_{f}
$$

to the calculated neutron multiplication factor values, $k_{\infty}$. Here, $T_{f}$ is the fuel temperature, and the parameters $a, b$, and $d$ are obtained by a (least square) regression method. The (lattice cell) reactivity is defined as $\rho_{\infty}=1.0-1.0 / k_{\infty}$. Then, the Doppler reactivity coefficient is calculated as:

$$
\begin{aligned}
\frac{\partial \rho_{\infty}}{\partial T_{f}} & =\frac{1}{k_{\infty}^{2}} \frac{\partial k_{\infty}}{\partial T_{f}} \\
& \approx \frac{1}{\left(a+b T_{f}^{1 / 2}+d T_{f}\right)^{2}}\left(d+b / 2 T_{f}^{1 / 2}\right) .
\end{aligned}
$$

Three different bundle configurations were used to investigate the impact of the DBRC scattering kernel for different fuel temperatures $T_{f}$. The first case (case 1) is the reference configuration of the 37-element $\mathrm{D}_{2} \mathrm{O}$ cooled bundle. In the second case (case 2), a voided bundle was investigated by reducing the coolant density to $1 / 1,000$ of the reference case. The third case (case 3 ) used pure $\mathrm{H}_{2} \mathrm{O}$ instead of $\mathrm{D}_{2} \mathrm{O}$ as coolant. The moderator outside of the fuel bundle is always $\mathrm{D}_{2} \mathrm{O}$ of high purity. In the third case, the natural Uranium $\mathrm{UO}_{2}$ fuel is replaced by $0.9 \mathrm{wt} \%{ }^{235} \mathrm{U}$ enriched $\mathrm{UO}_{2}$ fuel.

Figure 2 shows the criticality values $k_{\infty}$ as a function of the fuel temperature for the three cases described, where the results obtained with both the standard MCNPX and modified versions of MCNPX with DBRC are plotted. (The standard uncertainty of $k_{\infty}$ is $\Delta k= \pm 6 \mathrm{pcm}$ in all cases for all $T_{f} ; 90$ million active histories and 3,000 cycles were used.) Reasonably, the impact of the DBRC model becomes noticeable with increased temperature - the actual temperature dependent differences between the two models are given in Fig. 3. Above $800 \mathrm{~K}$ the effect of the DBRC model is significant $(\approx 50 \mathrm{pcm}=0.5 \mathrm{mk})$ and at high temperatures it reaches $100-150 \mathrm{pcm}(=1.0-1.5 \mathrm{mk})$. As the differences in $k_{\infty}$ shown in Fig. 3 are almost the same for the cooled and voided cases (cases 1 and 2), there is no impact of the DBRC on the coolant void reactivity $\left(\mathrm{CVR}_{\infty}=\rho_{\infty}\right.$ (voided $)-$ $\rho_{\infty}($ cooled $\left.)\right)$ of the 37 -element CANDU bundle with fresh 


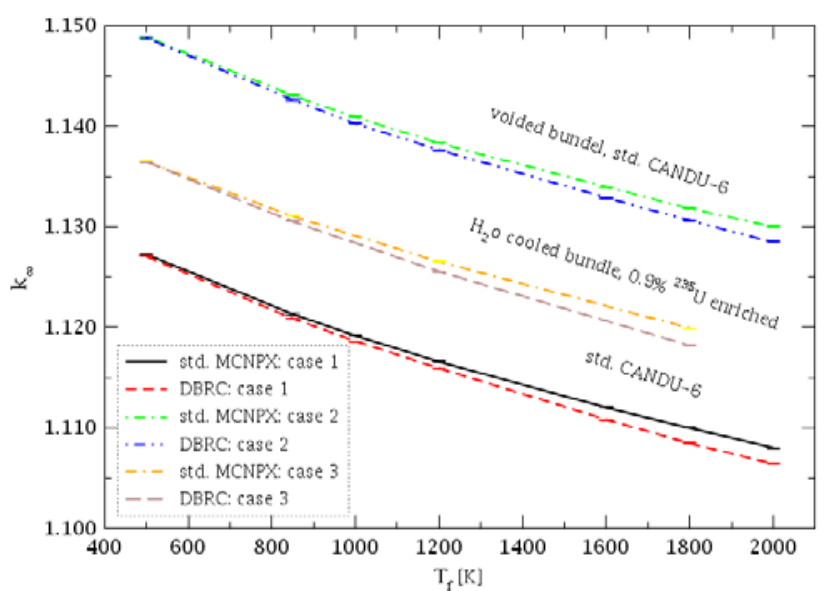

Fig. 2 Criticality of a 37-element fuel bundle calculated with std. MCNPX and MCNPX DBRC for different fuel temperatures $T_{f}$ and different bundle configurations

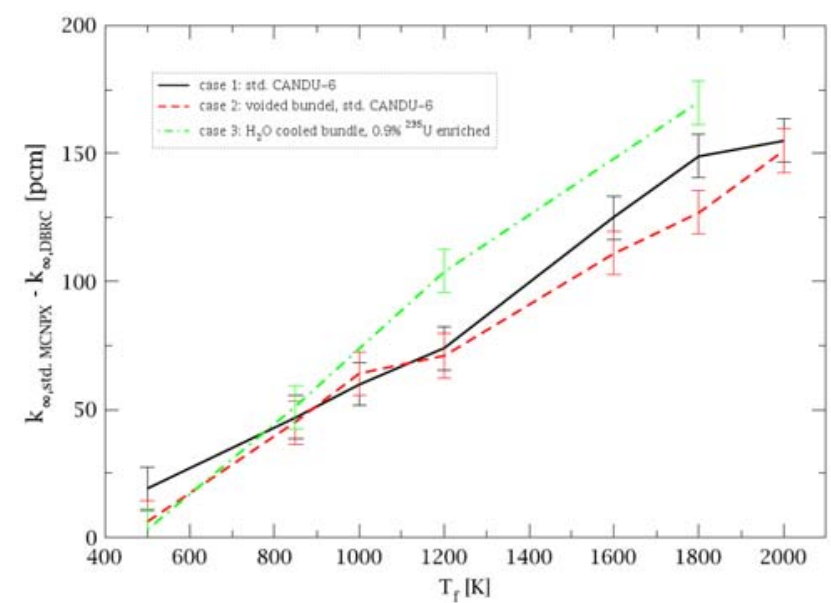

Fig. 3 Criticality difference of a 37-element fuel bundle calculated with std. MCNPX and MCNPX DBRC for different fuel temperatures $T_{f}$ and different bundle configurations

clean fuel. For example, the difference in $\mathrm{CVR}_{\infty}$ is less than $10 \mathrm{pcm}(=0.1 \mathrm{mk})$ near the design conditions.

The Doppler reactivity coefficient was calculated based on Eq. (6) (see Fig. 4). The insertion of the DBRC model increases the strength of the Doppler effect steadily with increasing temperature. At very high fuel temperatures a decrease in the Doppler reactivity coefficient is up to $11 \%$ (Fig. 5) in comparison with the standard MCNPX model.

In summary, this analysis shows that the DBRC effect is similar for all tested options, with a slight increase in the importance of the resonance scattering kernel for $\mathrm{H}_{2} \mathrm{O}$ coolant. We assume that this negative impact on the Doppler reactivity coefficient (3 to $10 \%$ for fresh clean fuel) can propagate to negative changes of the same order of magnitude in the CANDU power coefficient of reactivity (PCR) obtained in full core analysis. ${ }^{17)}$ However, in comparison with the light water reactors, the impact of DBRC on criticality and the Doppler effect in the heavy water CANDU cells is considerably lower, about $60 \%$ less for the criticality values and $30 \%$ less for the Doppler effect. ${ }^{5)}$
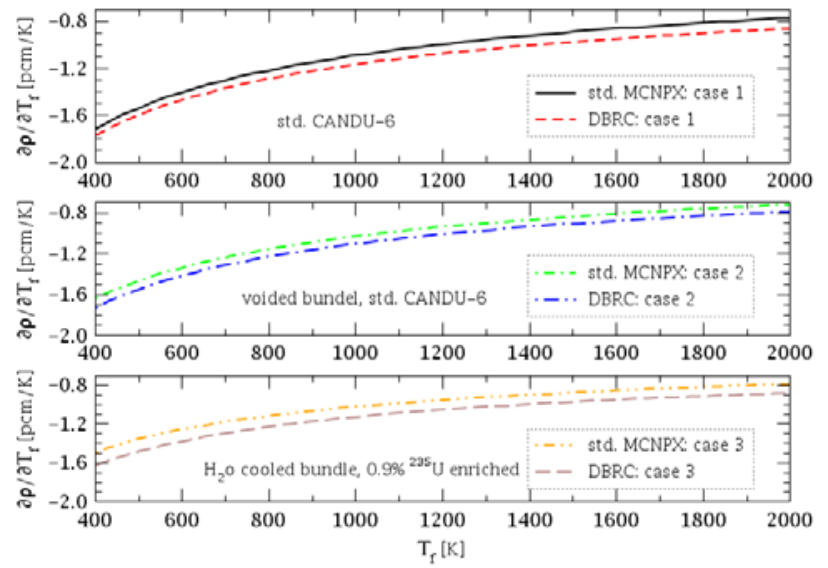

Fig. 4 Doppler reactivity coefficient of a 37-element fuel bundle calculated with std. MCNPX and MCNPX DBRC for different fuel temperatures $T_{f}$ and different bundle configurations
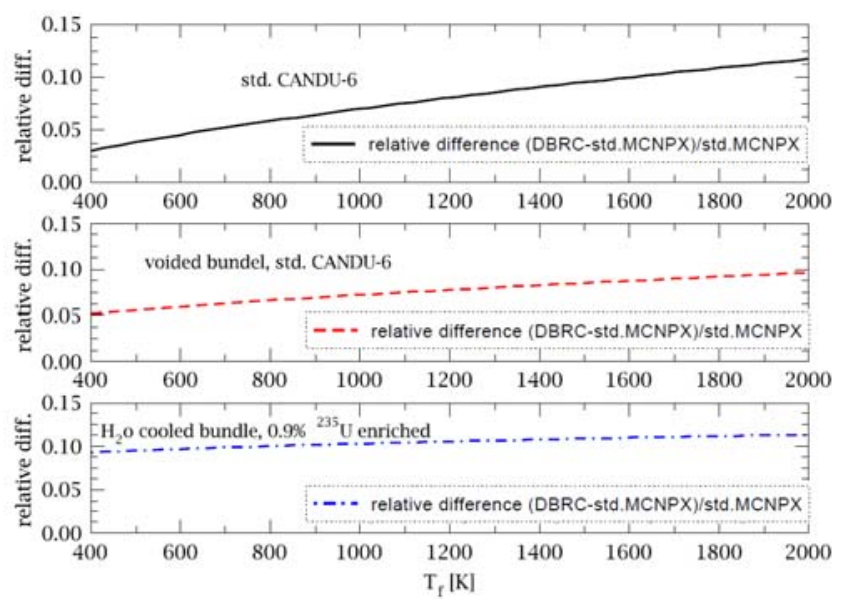

Fig. 5 Relative difference of the Doppler reactivity coefficient of a 37-element fuel bundle calculated with std. MCNPX and MCNPX DBRC for different fuel temperatures $T_{f}$ and different bundle configurations

\section{Sensitivity Analysis of the Resonant Scattering Kernel Effect Versus the Lattice Cell Pitch}

In view of the innovative concepts under study for the CANDU-type reactors, ${ }^{16,19)}$ a complementary study was performed. The reference 37 -element bundle pitch of $28.575 \mathrm{~cm}$ was reduced to $24.0 \mathrm{~cm}$ and $20.0 \mathrm{~cm}$. Because an $\mathrm{H}_{2} \mathrm{O}$-cooled bundle option is being considered for the new CANDU-type reactors, the 37-element $\mathrm{H}_{2} \mathrm{O}$-cooled bundle was taken as a reference (see Section III above). The criticality $\left(k_{\infty}\right)$ as a function of the fuel temperature was calculated as before with the standard MCNPX code and afterwards with the improved DBRC version. The decrease of criticality with decreasing lattice pitch is depicted in Fig. 6. As far as the DBRC effect is concerned, the decrease in lattice pitch enhances the importance of the DBRC treatment by up to $100 \%$ (Fig. 7), reaching values of $\approx 300 \mathrm{pcm}$, which are of the same order as for the light water reactors. This could be attributed to a shift of the neutron flux spectrum towards the resonance energy range accompanying the reduced moderation. 


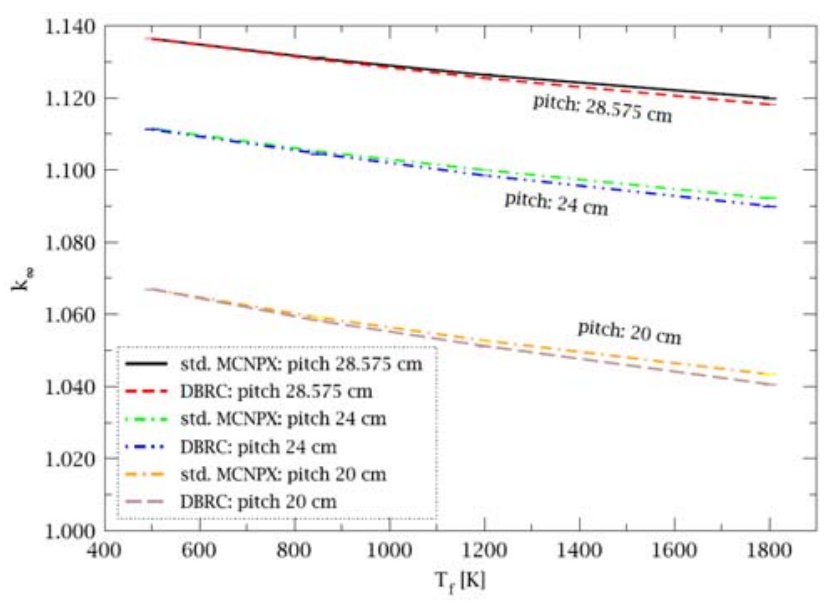

Fig. 6 Criticality of a light water cooled 37-element fuel bundle calculated with std. MCNPX and MCNPX DBRC for different fuel temperatures $T_{f}$ and different lattice cell pitch values

\section{Conclusion}

The impact of consistent Doppler broadening of the scattering kernel and the (integral) scattering cross sections of ${ }^{238} \mathrm{U}$ was investigated for CANDU-type fuel lattice cells. The scattering kernel was broadened by applying the DBRC method within the MCNPX code, and tested by comparison with the regular MCNPX method. The impact of DBRC on the criticality and Doppler reactivity coefficient of the heavy water moderated/heavy water cooled CANDU fuel bundles is small, but noticeable, near the design conditions (the decrease in $k_{\infty}$ is about $50 \mathrm{pcm}=0.5 \mathrm{mk}$ ). The magnitude of the DBRC impact increases with an increase in fuel temperature. We found a small negative impact on Doppler reactivity coefficient (3 to $10 \%$ ) and almost no impact on the coolant void reactivity $(<10 \mathrm{pcm})$ for the standard 37-element bundle with fresh clean fuel. When changing coolant from heavy water to light water and reducing the lattice pitch, the DBRC impact increases because the neutron spectrum has more weight in the resonance energy region.

\section{Acknowledgment}

The authors would like to thank M. Milgram, K. Kozier, and D. Altiparmakov for their interest, valuable suggestions and discussions that were very helpful for this study.

\section{References}

1) W. Rothenstein, R. Dagan, "Ideal gas scattering kernel for energy dependent cross-sections," Ann. Nucl. Energy, 25, 209222 (1998).

2) R. E. MacFarlane, D. W. Muir, The NJOY Nuclear Data Processing System, Version 91, LA-12740-M, Los Alamos National Laboratory (LANL) (1994).

3) W. Rothenstein, "Proof of the formula for the ideal gas scattering kernel for nuclides with strongly energy dependent scattering cross sections," Ann. Nucl. Energy, 31, 9-23 (2004).

4) R. Dagan, "On the use of $S(\alpha, \beta)$ tables for nuclides with well pronounced resonances," Ann. Nucl. Energy, 32, 367-377 (2005).

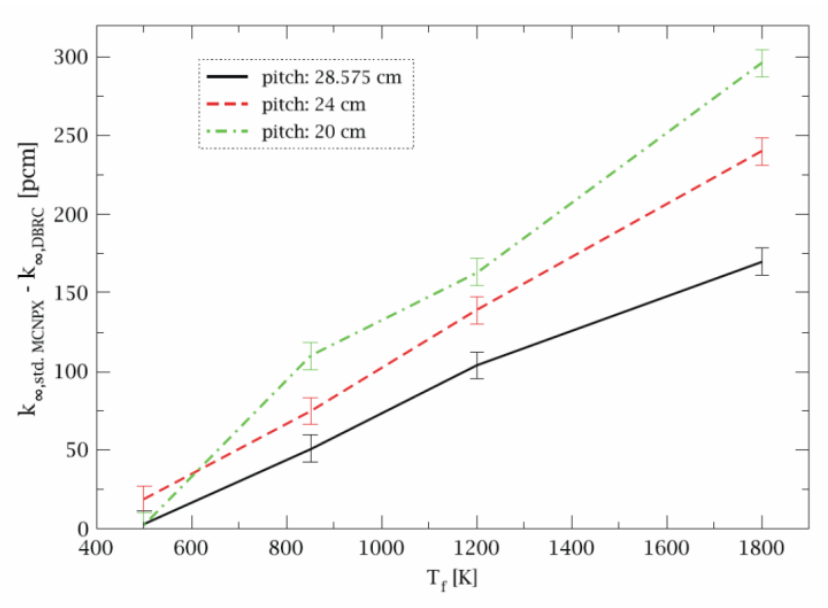

Fig. 7 Criticality difference of a light water cooled 37-element fuel bundle calculated with std. MCNPX and MCNPX DBRC for different fuel temperatures $T_{f}$ and different lattice cell pitch values

5) R. Dagan, C. H. M. Broeders, "On the effect of Resonance dependent Scattering-kernel on Fuel cycle and inventory," Proc. PHYSOR-2006, Sep. 10-14, 2006, Vancouver, Canada (2006), [CD-ROM].

6) B. Becker, R. Dagan, C. H. M. Broeders, G. Lohnert, "Improvement of the Resonance scattering treatment in MCNP in view of HTR calculations", Ann. Nucl. Energy, 36, 281-285 (2009).

7) B. Becker, R. Dagan, C. H. M. Broeders, "Proof and implementation of the stochastic formula for ideal gas, energy dependent scattering kernel," Ann. Nucl. Energy, 36, 470-474 (2009).

8) W. Rothenstein, "Neutron Scattering Kernels in Pronounced Resonances for Stochastic Doppler Effect Calculations," Ann. Nucl. Energy, 23, 441-458 (1996).

9) Y. Danon, E. Liu, D. Barry, T. Ro, R. Dagan, "Benchmark Experiment of Neutron Resonance Scattering Models in Monte Carlo Codes," Proc. International Conference on Mathematics, Computational Methods and Reactor Physics (M\&C 2009), May 3-7, 2009, Saratoga Spring, USA (2009), [CD-ROM].

10) X-5 Monte Carlo Team, MCNP-A General Monte Carlo N-Particle Transport Code, LA-UR-03-1987, Los Alamos National Laboratory (LANL) (2003).

11) D. B. Pelowitz, MCNPX $X^{T M}$ User's Manual, Version 2.5.0, LACP-05-0369, Los Alamos National Laboratory (LANL) (2005).

12) E. P. Wigner, J. E.Wilkins, Effect of the temperature of the moderator on the velocity distribution of neutrons with numerical calculations for $H$ as moderator, AECD-2275, Oak Ridge National Laboratory (ORNL) (1944).

13) D. Emendö. Emend. H. Hndker, Theorie der Kernreaktoren, Wissenschaftsverlag (1982), [in German].

14) M. M. R. Williams, The Slowing Down and Thermalization of Neutrons, North-Holland Publishing (1966).

15) R. Dagan, B. Becker, Implementation of the Resonant Scattering Kernel in Monte Carlo Codes, NEA, http://www.nea.fr/dbforms/data/eva/evatapes/jeff_31/Resonant -Scattering-Kernel-Dagan/ (2010).

16) D. F. Torgerson, B. A. Shalaby, S. Pang, "CANDU technology for generation III+ and IV reactors," Nucl. Eng. Design, 236, 1565-1572 (2006).

17) M. A. Lone, "Fuel Temperature Reactivity Coefficient of a CANDU Lattice - Numerical Benchmark on WIMS-AECL (2-5d) Against MCNP," Proc. Twenty Second Annual Confe- 
rence of the Canadian Nuclear Society, June 10-13, 2001, Toronto, Canada (2001), [CD-ROM].

18) K. S. Kozier, "Assessment of CANDU Reactor Physics Effects Using a Simplified Whole-Core MCNP Model," Proc. PHYSOR-2002, Oct. 7-10, 2002, Seoul, Korea (2002), [CD-
$\mathrm{ROM}]$.

19) A. Buijs, M. Bonechi, M. Boubcher, P. S. Chan et al., "The Physics Design of the Advanced CANDU Reactor," Proc. PHYSOR-2008, Sept. 14-19, 2008, Interlaken, Switzerland (2008), [CD-ROM]. 